\title{
Non-defensible Middle Ground for Experimental Realism: Why We Are Justified to Believe in Colored Quarks*
}

\author{
Michela Massimi ${ }^{\ddagger}$
}

Experimental realism aims at striking a middle ground between scientific realism and antirealism, between the success of experimental physics it would explain and the realism about scientific theories it would supplant. This middle ground reinstates the engineering idea that belief in scientific entities is justified on purely experimental grounds, without any commitment to scientific theories and laws. This paper argues that there is no defensible middle ground to be staked out when it comes to justifying physicists' belief in colored quarks, and that experimental realism shifts, under analysis, into scientific realism.

1. Introduction. Entity realism emerged at the beginning of the $1980 \mathrm{~s}$ as a refreshingly new position in the philosophical stand-off between scientific realism and anti-realism. The publication of Bas van Fraassen's book The Scientific Image (1980) issued an empiricist challenge to the traditional realist view. From an empiricist viewpoint, belief in the truth of scientific theories seemed a gratuitous addition to the more modest belief in the empirical adequacy of the theory. And by dispensing with the full truth of scientific theories, the truth of the theoretical claims about unobservable scientific entities was dispensed with altogether.

The novelty of entity realism consisted precisely in addressing van Fraassen's challenge without falling back on scientific realism. Among the varieties of entity realism, Ian Hacking's experimental realism occupies an

*Received January 2003; revised August 2003.

$\dagger$ To contact the author write to Girton College, Cambridge, CB3 0JG, UK; e-mail: mm483 @)cam.ac.uk.

$\ddagger$ I am very grateful to Peter Lipton and Michael Redhead for insightful comments and suggestions on earlier drafts of this paper. I have much benefited from stimulating comments made by Steve Clarke, Mauro Dorato, Mary Leng, Robert Nola, and Mark Sprevak. I thank Emanuele Leonardi (CERN) for friendly help with the reference of the CDHS experiment and color confinement. The challenging and constructive criticisms of two anonymous referees helped me to clarify the ideas here exposed.

Philosophy of Science, 71 (January 2004) pp. 36-60. 0031-8248/2004/7101-0004\$10.00

Copyright 2004 by the Philosophy of Science Association. All rights reserved. 
important position. Hacking has famously argued that "engineering, not theorizing, is the proof of scientific realism about entities" $(1982,86)$. The majority of experimenters would be realists about entities but agnostic about theories. Realism about entities combines nicely with anti-realism about scientific models, which are simply "tools for thinking and calculating ... essential for writing up grant proposals to obtain further funding" but nothing more than rules of thumb used to get things done (Hacking $1982,73)$. On this view, realism is licensed by the causal role scientific entities play in experimental situations: as long as we can manipulate and intervene with them in a lab to create new phenomena, we are justified to believe in their existence as the causal entities at work in the observed effects. For instance, in the polarizing electron gun PEGGY II electrons are manipulated and sprayed to create parity violating phenomena in weak neutral currents; whence the intriguing slogan "if you can spray them, they exist."

Yet, the criteria of manipulation and intervention do not seem to capture all scientific entities, and for instance "the experimenter cheerfully regards neutral bosons as merely hypothetical entities, while electrons are real" (Hacking 1982, 76). A change of status from hypothetical to real entity occurred with the neutrino, which, originally hypothesised in 1931 to account for $\beta$ decay, "is now routinely used to examine other things" (Hacking 1982, 85). The controversial nature of this change of status from hypothetical to real entity, related as it is to the contingent availability of an experimental set-up and technique, has been noted by Resnik, who labels Hacking's argument a "case-by-case" realism, where "if science is able to use its posits as tools for experimentation, then the posits are real. If not, then they are merely hypothetical. An implication of this view is that one can be realist about electrons but not about quarks or weak neutral currents" (Resnik 1994, 401), nor, needless to say, about astrophysical entities. And on this last point, Dudley Shapere (1993) has pointed out that there are two different ways of intending the verb "to use," a strong one (i.e., to manipulate) and a weak one (to employ), and while astrophysical entities do not meet the first, as Hacking complains, they definitely meet the second one, and as such we can remain realists about them.

Resnik has raised an important objection to Hacking's position: "the experimental realist can only have knowledge about theoretical entities if she assumes that the theories which describe those entities are at least approximately true; and ... that experimentation is not nearly as theory-free as Hacking maintains" (Resnik 1994, 395). The issue of theory-free experimentation concerns experimental realism no more than entity realism in general. Musgrave (1996), for instance, has emphasised the dichotomy between realism about entities and realism about theories by wittily portraying entity realists as believing in entities such as-say- 
hobgoblins without endorsing any belief about what hobgoblins do or what they are like. More recently, Steve Clarke (2001) has offered a defence of entity realism against this "unfortunately popular caricature" (704): entity realists do commit themselves to theories. Yet, this commitment is restricted to lower-level phenomenological laws and very specialised principles and it does not extend to fundamental laws or to full-blown theories. ${ }^{1}$

Following up on Resnik's objection, in this paper I argue that, to be warranted in being a realist about some entities, the experimental realist must take a position that commits her to believe in the scientific theory concerning these entities. I follow an argumentative route different from Resnik, who tackled Hacking's theory-free experimentation by focussing on his reliance on Putnam's semantic theory. ${ }^{2}$ My argumentative route does not pass through theories of reference, but rather through an appropriate version of the underdetermination argument.

The problem of empirical equivalence and underdetermination may arise in fact in experimental realism no less than in scientific realism. There might be more than one potential causal entity at work behind the observed phenomena (empirically equivalent entities), so that the choice among them would be underdetermined by evidence. And - as I shall arguethere is no way of distinguishing between empirically equivalent entities unless we have already distinguished and chosen between their rival scientific theories. In other words, we cannot help committing ourselves to full-blown theories to select the potential causal entity from among the rivals. The gap between experiment and theory is not as large as experimental realists depict it. And once it is bridged, it is bridged both for phenomenological and for fundamental laws.

In Section 3, I illustrate this argument with the historical case study of how physicists came to believe in colored quarks, and why this belief supplanted their previous belief in partons as potential constituents of

1. Clarke's defence of entity realism follows up on Cartwright's argument that causal explanations would justify us to believe in the entity as the putative cause of the phenomena at issue, without being also realist about scientific theories and fundamental laws of nature. In this paper I do not address Cartwright's argument, based on the difference between causal and theoretical explanations, and I focus rather on experimental realism and its engineering criterion of manipulation.

2. Resnik has attacked Hacking's experimental realism precisely on this issue by pointing out that "while [Putnam's semantic theory] establishes the cogency of Hacking's metaphysical part of theory-free entity realism, it does not go very far in supporting the epistemological aspect of this view. Theory-free entity realism is on epistemologically solid ground - it can give us knowledge - only if we know that the entities to which we refer are natural kinds; that is, we know that they are fundamental parts of the world's causal structure" (Resnik 1994, 407). 
nucleons, despite the fact that both partons and quarks could account for the known properties of nucleons and the same experimental data could be retrieved by assuming either partons or quarks. The evidence in favour of colored quarks came from extraordinarily theory-loaded experiments on deep inelastic lepton-hadron scattering that cannot be interpreted at all unless we have already committed ourselves to quantum chromodynamics (QCD) and to its entire theoretical arsenal.

Evidence and theory are inextricably intertwined in grounding our belief in colored quarks, and there is no distinctive middle ground to be staked out. The middle ground of experimental realism resembles rather the edge of a cliff where one is provisionally about to slide either to the anti-realist camp, or-as I shall argue here - to the realist one. Maybe the advantage of occupying a middle ground consists precisely in sitting on the edge of two different camps. Yet, if in the end I find myself in the realist camp trying to persuade experimental realists to join the group, ${ }^{3}$ this should be read with an eye towards improving our understanding of an open issue, whose importance Hacking's stimulating and intriguing philosophical position has helped us to appreciate.

\section{The Problem of Empirical Equivalence Between Putative Entities.} Empirical equivalence and underdetermination have traditionally been championed by anti-realists against realism about scientific theories. If there are two or more theories able to entail the same empirical consequences $^{4}$ (empirically equivalent theories), the choice among them is underdetermined by evidence, and hence belief in one theory rather than another is arbitrary and unfounded. Friends and foes of underdetermination have engaged in interminable debates. The former insist on the possibility of constructing infinitely many empirically equivalent theories via algorithms (Kukla 1996). The latter have rejoined that underdetermination is based on a simplified conception of science typical of hypotheticodeductivism (Laudan and Leplin 1991).

As every realist, I am inclined to reject underdetermination and empirical equivalence. Yet, it is important to consider carefully the challenge that underdetermination poses. In this paper I want in particular to focus on a generally overlooked entity-version of the underdetermination argument

3. Of course, a detailed investigation of the reasons for choosing scientific realism rather than experimental realism would require an analysis of all the objections that experimental realists, and entity realists more in general (for instance, Cartwright 1983), have raised against scientific realists, as well as the replies to these objections (for instance, Franklin 1996). But this will lead me far astray from the scope and purpose of this paper, which is to highlight some problematic aspects of the engineering criterion of experimental realism.

4. For an analysis of underdetermination in its entailment version see Laudan 1990. 
that arises in the specific territory of experimental realism (and entity realism, more in general). This runs as follows: there might be two or more putative entities (say, $x$ and $y$ ) potentially causing the same experimental evidence (empirically equivalent entities). And if rival entities can potentially cause the same evidence, the choice among them is underdetermined, and it becomes questionable whether we see experimental evidence as vindicating the existence of the entity $x$ or $y$. This entity-version is a suitably weak form of what Laudan (1990) has portrayed as the nonuniqueness thesis of underdetermination. Paraphrasing Laudan, for any entity $x$ and evidence $E$ potentially caused by $x$, there may be a rival entity $y$ that could cause $E$ as well as $x$.

I shall here play the role of the devil's advocate for this entity-version of empirical equivalence in order to argue that sheer experimental evidence by itself does not tell us which entity is at work in the observed phenomena; for that we must commit ourselves to a full-blown scientific theory. In other words, there is no privileged experimental ground for entity-choice because empirical equivalence may arise at the level of putative entities. So the only way of solving the entity-version of empirical equivalence consists in first solving the theory-version. Experimental realists face then the following dilemma: either they resign themselves to the problem of empirical equivalence between putative entities, or they solve it at the cost of a substantial theoretical commitment. To put it differently, either they cannot be realists about entities, or they will also be realists about scientific theories.

Steve Clarke has discussed the problem of empirical equivalence in his recent defence of entity realism. Although he presents the problem as emerging from the inference to the best explanation framework that I do not consider here, still the way he poses the objection and his reply to it are worth quoting in some detail:

It seems logically possible, for example, that the observed and experienced effects that we explain by appealing to the existence of a single entity, the electron, might actually be caused by a cluster of microscopic particles that are invariably joined together.... And it seems that we have no non-arbitrary way of choosing between belief in the electron, as against any of these possibilities, without appealing to pragmatic considerations. My response to this line of objection is that the alleged different possible entities either turn out, on closer inspection, to be different theories about the composition, or the nature, of the same entity, or it turns out that we have not interacted with the entity that we are claiming exists, and that what has happened is a case of an experiment that, unbeknownst to us, has not been successful.... If we were to refrain from referring to particular entities, on the grounds that these might possibly be divisible, then it appears that we could 
never refer to any entities, as it appears that we can never be in a position to be certain that any given entity is not divisible. I take it that this is an unacceptable outcome. (Clarke 2001, 719-720)

Clarke is right to reject that outcome. However, the real challenge of the objection lies elsewhere. Being amenable to further division is not by itself a difficulty; but it may become a difficulty for entity realists in a very subtle way. Clarke's example of the electron is a favourable one for entity realists, since electrons are not divisible entities, according to the standard model. But consider instead hadrons - protons, say. ${ }^{5}$ Protons are clusters of microscopic entities, and there might be another cluster of rival entities equally accounting for the known properties of protons. The same entity (proton) can be regarded as consisting of different sub-entities. Empirical equivalence may arise at the level of these rival clusters of sub-entities, not in the comparison of the proton with the cluster itself, as Clarke's reply suggests.

Indeed, looking back at the history of quantum chromodynamics (QCD), protons (and neutrons) were originally regarded as clusters of partons, and later as clusters of quarks. Partons and quarks are not just different theories of one and the same entity we have unequivocally interacted with in the lab. Rather, they are different entities endowed with different dynamic properties (i.e., to stick to a terminology dear to entity realists, endowed with different causal powers/capacities/ dispositions to act in a certain way) that nonetheless could equally account for the known properties of nucleons.

Before plunging into the fascinating world of partons and quarks in the next section, a preliminary objection needs to be considered. An experimental realist would surely refrain from putting on a par the entityversion and the theory-version of empirical equivalence. She would insist that - despite similarities - the entity-version of empirical equivalence is crucially different (and less threatening) than the theory-version. In fact, while it is logically possible for rival theories to entail the same phenomenon, it is not similarly the case that two rival entities can both cause the same phenomenon. In other words, the relationship between the

5. According to the standard model, elementary particles divide into leptons and hadrons. Leptons (from the ancient Greek word "light") are particles with light masses such as electrons, neutrinos, and muons. Hadrons include mesons (again from the Greek, particles with medium mass) such as pions and kaons, and baryons (particles with heavy mass) such as nucleons (protons and neutrons) among others. The current classification of particles in leptons, mesons, and baryons does not necessarily reflect the light/heavy distinction about masses (i.e., among the leptons, the tau has a mass of $1777 \mathrm{MeV}$, almost twice the mass of baryons such as protons and neutrons). A more precise definition of this classification is offered by QCD, as clarified in Section 3. 
description of a phenomenon and empirically equivalent theories is logical, whereas the relationship between a phenomenon and the entities that can produce that phenomenon is causal. This distinction is orthogonal to the distinction between descriptivist theories of reference and causal theories of reference, where typically scientific realists are inclined to the first and experimental realists to the second: realism about entities is ultimately warranted by a successful causal reference-fixing procedure. ${ }^{6}$ Theories (and theoretical descriptions) come and go, while entities are causally fixed once and for all.

Thus, an experimental realist would not be concerned about different ways of conceiving the proton's constituents as quarks or partons so long as the observed phenomena could have been produced by either. In other words, her theory-free attitude towards experimentation would lead her to say: "There is some entity inside the proton that produces the observed effects. These entities (no matter how they are theoretically characterised by the parton model) have certain causal powers/capacities/dispositions to produce certain effects. I am realist about these entities having these causal powers/capacities/dispositions." If we later discover that among the observed effects, there are some which are incompatible with the parton model, the experimental realist can still retain realism about the entities that produce the observed effects, regardless of whether these are no longer characterised as partons (in the sense of the parton model), but rather as quarks (in the sense of QCD).

Working through the history of lepton-hadron scattering experiments illuminates this theory-free stance on experimentation, and cases of theoretical divergence such as that between the parton model and QCD are excellent tools for exploring the relationship between the theory-version and the entity-version of empirical equivalence. The next sections clarify how this relationship works out, as well as the parallelism between the two versions. The theory-version of empirical equivalence is based on the possibility of rival theories to entail the same phenomena; the entity-version is based on the possibility of rival entities to cause the same phenomena (where notice that potential rather than actual causation is here at stake). Partons and quarks were rival potential causes for the known properties of nucleons. The main point of my argument is that whenever we have prima facie rival potential causes for the same phenomena, in order to distinguish between them and to determine what entity-with-causal-powers has actually produced the observed effects, we must in the end rely on the description of

6. Despite the debt to Putnam's meaning of 'meaning' (Putnam 1975), Hacking himself discussed some major difficulties in the case of bifurcating concepts such as acidity, or nonentities such as caloric, or names stolen such as "meson" to the extent of urging a theory of reference that could improve on Putnam (Hacking 1983, chap. 6). 
what causal powers/capacities/dispositions an entity is to have so as to produce the observed effects. This description is given by a scientific theory; and rival theories give rival descriptions. ${ }^{7}$ Only by choosing between different descriptions, and hence by committing ourselves to one theory rather than to another, can we identify what entity is at work behind the experimental phenomena.

Scientific realism traditionally contends that we are justified to believe in the theoretical claims of our scientific theories, and that we must read these claims literally, i.e., we must believe that the entities postulated by the theories exist and have the properties defined by the theories. ${ }^{8}$ This is a typical top-down approach: from theory-realism to entity-realism, where belief in the existence of scientific entities is justified on theoretical grounds (insofar as they are postulated by the theories we believe to be true). Here I want rather to suggest the following bottom-up approach to scientific realism, starting from the experimental ground typical of experimental realism: the decisive evidence that warrants us to believe in the existence of some entities with certain properties (over rival empirically equivalent ones) is tout court the same evidence that warrants us to believe in the scientific theory concerning these entities, rather than in a rival one.

In the next sections I retail a little particle physics in presenting the rival parton and quark models of hadrons. Some mathematical details are necessary to show in what sense partons and quarks were empirically equivalent, in what respect they were not, and how physicists could discriminate between the two in favour of quarks. As it will become evident, we cannot help believing in QCD in order to believe that colored quarks are the entities causally at work in deep inelastic scattering experiments.

3. Partons or Quarks? According to QCD, hadrons are made up of quarks. Quarks have different physical properties such as charge, isospin, ${ }^{9}$ and

7. Rival empirically equivalent theories typically give different descriptions, and the qualification "empirically equivalent" is just due to their being equally able to somehow entail to same phenomena (via their respective descriptions). This is usually achieved by postulating different mechanisms, one of which is an empirically equivalent surrogate for the correct one (see, for instance, Massimi, forthcoming, for an analysis of rival empirically equivalent spectroscopic theories in the early 1920s).

8. This view is paradigmatically expressed by Wilfred Sellars: "to have good reason for holding a theory is ipso facto to have good reason for holding that the entities postulated by the theory exist" (Sellars 1962, 97).

9. Isospin $I$ is a symmetry requirement that quarks satisfy: it is invariance under $\mathrm{SU}(2)$ transformation, in the language of group theory. For example, particles belonging to an isospin doublet (e.g., protons and neutrons) can be regarded as different states of one and the 
strangeness. ${ }^{10}$ The first three kinds (or "flavors") of quarks to be discovered were the up (u), down (d), and strange (s) quarks. Mesons consist of a quarkantiquark pair q $\overline{\mathrm{q}}$, while baryons consist of a quark triplet qqq (e.g., protons are uud triplets, neutrons are udd triplets). Same flavor quarks (say, the two u quarks in protons) are differentiated by having different "colors": red (r), blue (b), or green (g). The carriers of colors are massless particles called "gluons," and just as intermediate vector bosons are the carriers of the weak interaction, photons are the carriers of the electromagnetic force, similarly in the standard model gluons are the carriers of the strong interaction that binds quarks together to form baryons and mesons. Quarks exchange gluons and change accordingly their color (e.g., a u blue quark becomes $\mathrm{u}$ red by emitting a gluon $\mathrm{G}_{\mathrm{b}_{\overline{\mathrm{F}}}}$ ). Quarks are invariant under a particular transformation in group theory, the so-called $\mathrm{SU}(3)$ color symmetry. Without lingering on mathematical details, it suffices here to say that this symmetry constraint on the color wavefunctions results in the so-called color confinement. As in Newton's theory of light, colors are confined in the spectrum of the colorless light, similarly in QCD only "colorless" singlet states can exist as free particles. And colorless singlet states are precisely quark-antiquark pairs (mesons) or quark triplets (baryons). But since gluons and quarks carry net color, they are confined inside mesons and baryons and they cannot exist as free particles. ${ }^{11} \mathrm{~A}$ free quark is prohibited in principle, since it would already have combined with other quarks or antiquarks to form new hadrons.

How did physicists arrive at this picture of hadrons as consisting of quark triplets? In the 1960s experimental evidence suggested that the inner structure of hadrons such as protons and neutrons was not uniform, but consisted of point-like entities (originally called "partons") whose nature and properties were investigated by particle physicists in the following ten years. Deep inelastic scattering was the typical experimental technique designed to investigate the inner structure of nucleons. The experimental

same entity (namely, the isospin) 'spinning'-so to speak-in different directions of an abstract three-dimensional space.

10. Strangeness $S$ was originally introduced by analogy with the conservation of charge in electron-positron annihilations as a property that was conserved in the strong interactions and violated in the weak interactions.

11. "Color confinement is the hypothesis that hadrons can only exist in states, called color singlet states, which have zero values for all color charges; while quarks which have nonzero color charges can only exist confined within them.... This explains why hadrons have integer electric charges while fractionally charged combinations like qq ... are forbidden, in accordance with experimental observation" (Martin and Shaw 1999, 142). In other words, since $(1 / \sqrt{3})(\bar{r} \bar{r}+g \bar{g}+b \bar{b})$ is a color singlet, $q \bar{q}$ entities (i.e., mesons) can exist as free particles. Similarly, $(1 / \sqrt{6})(\mathrm{rgb}-\mathrm{grb}+\mathrm{gbr}-\mathrm{bgr}+\mathrm{brg}-\mathrm{rbg})$ is also a color singlet state, then qqq entities (i.e., baryons) can exist as free particles. Other quark bound states whose total color charge does not add up to zero do no exist as free particles. Like quarks, gluons 
data so obtained were originally cast within the so-called parton model (Bjorken and Paschos 1969; Feynman 1972), and only later reinterpreted in the light of the colored quark model.

Historically, quarks had already been introduced by Gell-Mann (1964) to account for the known properties of hadrons within a phenomenological model called the "eightfold way" (Gell-Mann 1961; Ne'eman 1961). ${ }^{12}$ In order to accommodate some anomalous hadronic spectra, soon it was suggested that quarks should have a further degree of freedom, the color (Han and Nambu 1965). The colored quark model was then originally developed on mainly theoretical grounds, and independently of the deep inelastic scattering experiments that — more or less at the same time-were giving evidence for partons. This explains the original reluctance of some physicists such as Feynman to endorse the quark theory.

However, as soon as experiments showed that the spin and the electric charges of partons were indeed the same as those of the quarks (see Section 3.2 below and Appendix 1), it seemed natural to identify partons with quarks. This identification was based on the empirical equivalence between partons and quarks as far as some relevant physical properties were concerned (spin, charge, number of constituents in baryons). And no wonder that in the physics literature of the early 1970s, physicists often used the double name "parton quark" and maintained an ambiguous language to describe their experimental findings.

Partons, however, could only be identified with quarks to a certain extent. According to the parton model, partons do not radiate gluons as colored quarks do, and therefore they do not exchange quantum-chromodynamic force. Partons behave as free particles inside nucleons, while

too do not exist as free particles because they are the carriers of net colors. Nonetheless gluon bound states whose total color charge is zero (such as gg, gggg, etc.) can in principle exist: they are called glueballs and recently some experimental evidence has been found for their existence (Close 1997). Notice that the expression "do not exist as free particles" does not mean that quarks or gluons cannot be knocked out from their bound states: indeed, they can and are typically knocked out in experimental situations. However, once knocked out, they immediately recombine with other quarks and gluons to form new particles, i.e., the experimentally observed hadronic jets.

12. The eightfold way was a successful model of classification for hadrons. Hadrons were gathered in supermultiplets sharing some important physical properties (baryon number, spin, parity), and the particles of each supermultiplet were regarded as different states of one and the same entity 'spinning' - so to speak - in different directions of an abstract, complex space (the space of unitary spin). The model turned out to be heuristically fruitful to predict the existence of new particles (e.g., the omega minus) but it did not explain why hadrons gathered so nicely into supermultiplets. For that reason, quarks as potential constituents of hadrons were introduced. By theoretically assigning to quarks spin-1/2, fractional charge, and other quantum numbers, the supermultiplets of the eightfold way arose naturally, and it became possible to explain the known properties of baryons and mesons. 
colored quarks are bound together by the strong interaction. Thus, the dynamic properties of partons differ remarkably from those of quarks. In this respect, partons and quarks can no longer be identified, and when experimental evidence for quantum-chromodynamic properties was found in 1979 (CDHS experiment, see Section 3.2 below and Appendix 2), physicists went on to disown partons. Quarks, originally introduced for mainly theoretical reasons, ended up 'stealing'-so to speak-their empirical support from partons, which were subsequently discarded as potential constituents of nucleons. The naïve parton model can still be found in particle physics textbooks as a historical curiosity belonging to the prehistory of the quark model.

The parton model was in vogue for almost ten years, because it turned out to be a good approximation to perturbative QCD whenever processes involved short-distance interactions. ${ }^{13}$ This offers a particularly interesting case study to investigate the empirical equivalence between the rival entities posited by the two models. Before going into the details of this case study, a ground-clearing remark on the nature of the deep inelastic lepton-hadron scattering experiments is necessary.

\section{1. 'Seeing' the Nucleon's Constituents. Lepton-hadron scattering} experiments consist in scattering a leptonic beam (electrons, muons, or neutrinos) off a hadronic target (say, protons or neutrons). Depending on the momentum transfer between the incident electron beam e, and the nucleon target (say, a proton $\mathrm{p}$ ), the two following situations occur:

1. At low momentum transfer $-q^{2}$ [where $-q^{2}=-\left(p-p^{\prime}\right)^{2}$ with $p$ and $p^{\prime}$ the momenta of the initial and final electron beams, respectively]: ep $\rightarrow$ ep (elastic scattering).

2. At high momentum transfer $Q^{2}$ the proton $\mathrm{p}$ breaks up into many particles $\mathrm{X}$ (where $\mathrm{X}$ is the set of hadrons allowed by the conservation laws): ep $\rightarrow \mathrm{eX}$ (deep inelastic scattering).

Deep inelastic electron-proton scattering experiments offered for the first time experimental evidence for the existence of inner constituents of nucleons. In physicists' language, the structure of nucleons could be 'seen' in this kind of experiments, where 'seen' is here used in a very liberal sense. In particle physics textbooks it is a commonplace to present scattering experiments as follows: "as $Q^{2}$ is increased ..., the photon [i.e., the incident leptonic beam] starts to 'see' evidence for the point-like

13. By the uncertainty relations, the higher the momentum of the incident leptonic beam in scattering experiments, the shorter the time scale of the parton interaction inside the nucleons so that the QCD fine structure constant $\alpha_{s}$ is small enough for perturbative QCD calculations to remain valid in the parton model. 
valence quarks within the proton" (Halzen and Martin 1984, 216). Needless to say, physicists can and typically do cheerfully talk of 'seeing' entities beyond the realm of observation, in a way that most philosophers would find unsatisfactory, given their ontological scruples. Dudley Shapere (1982), and Hacking himself (1983, chap. 10-11) have dedicated extensive and illuminating analyses to the concept of observation in physics, and I want to latch onto this debate to shed light on what physicists actually 'see' in lepton-hadron scattering experiments.

In order to investigate the sense in which physicists talk of 'seeing' unobservable entities, Shapere (1982) has analysed the 'observation' of the sun's hot core via solar neutrinos: solar neutrinos are collected in a disused underground mine filled with carbon tetrachloride, where a few of them will form radioactive nuclei and by counting the number of neutrinos subject to this reaction, useful information about the interior of the sun can be obtained. We can then 'see' the sun's hot core via solar neutrinos because information about the sun is received by an appropriate receptor, and it is transmitted from the sun to the receptor without interferences. As Hacking remarks:

Shapere notes that whether or not something is directly observable depends upon the current state of knowledge. Our theories of the workings of receptors, or of the transmission of information by neutrinos, all assume massive amounts of theory. ... Yet we must never fall prey to the fallacy of talking about theory without making distinctions. For example, there is an excellent reason for speaking of observation in connection with neutrinos and the sun. The theory of the neutrino and its interactions is almost completely independent of speculations about the core of the sun. It is precisely the disunity of science that allows us to observe (deploying one massive batch of theoretical assumptions) another aspect of nature (about which we have an unconnected bunch of ideas).... Shapere had a more philosophical purpose in his analysis of observing.... He notes that what counts as observation depends upon our theories of the world and of special effects.... I have wanted to insist on some of the more humdrum aspects of observation. A philosophy of experimental science cannot allow theory-dominated philosophy to make the very concept of observation become suspect. (Hacking 1983, 183)

So, physicists can cheerfully talk of 'seeing' the sun's core, given our current state of knowledge (massively at work in the solar neutrino experiments) so long as the batch of theoretical assumptions upon which this 'observation' relies is not mixed up with the very same facts under investigation (and with the theories about these facts we may have).

The solar neutrino experiment provides us with an interesting analogue of 'seeing' the nucleon's constituents in the lepton-hadron scattering 
experiments that I am going to present in the next section. As we can 'see' the sun's core, similarly we can somehow 'see' the nucleon's core. But what does 'seeing' mean in the experimental context of lepton-hadron scattering experiments?

The more accurate term for 'seeing' here is 'probing'. The nucleon's core is said to be 'probed' in deep inelastic lepton-hadron scattering experiments. Atoms can be probed, protons and neutrons can be probed, but the sun's core cannot be said to be 'probed'. Let us then try to understand what probing means by cashing out the difference between 'seeing' the sun's core and 'seeing' the nucleon's core.

Physicists can arguably claim to 'see' the sun's core by inferring its properties from data about solar neutrinos that are collected and analysed as described by Shapere. To this purpose, Hacking notices that "although the extent of neutrino manipulation is much less than electron manipulation in the PEGGY II experiment, here we are plainly using neutrinos to investigate something else" (Hacking 1982, 85). However, strictly speaking, solar neutrinos are not manipulated: they are not used to interfere or intervene in other entities. They are simply gathered so as to infer information about something else (the sun).

Physicists can arguably claim to 'see' quarks and gluons by probing the inner structure of nucleons (protons or neutrons) with leptonic (electrons or neutrinos) beams. In other words, they use leptonic beams to intervene in nucleon targets, and from the analysis of the scattering angles, crosssections, and other important features of the lepton-hadron scattering they can 'see' what the nucleon's constituents are.

In nuclear physics probing is the typical procedure to 'see' unobservable entities inside atoms. Probing is a form of manipulation: it is still based on the engineering idea of using some entities to interfere / intervene in others so as to create new phenomena (e.g., the Bjorken scaling-violating phenomena I shall describe in the next section). In this sense, probing is just as good as manipulation.

Yet, probing is a weaker form of manipulation than "spraying" entities at work in the electron-gun PEGGY II, because what actually creates the new phenomena, i.e., the putative causal entities at work behind these new phenomena, are not themselves directly manipulated (i.e., sprayed) in the probing procedure. In other words, the entities producing the new observed phenomena are not the leptons of the incident beam that physicists 'spray' against the nucleon target. Rather, they are some entities inside the nucleon target that cannot be extracted from it and sprayed. Given the color confinement mentioned in Section 3, quarks and gluons cannot exist as free particles, and as such they cannot be sprayed (a quark-gun is prohibited in principle). In this specific sense, probing is not as good as manipulation (if by "manipulation" we intend "spraying" entities). Yet, it is not worse than 
solar neutrino manipulation (where "manipulation" is intended in a very liberal sense as collecting solar neutrinos to infer information about the sun's core). And if experimental realists can countenance solar neutrinos as a form (albeit weaker than "spraying") of manipulation, then a fortiori they can countenance probing nucleons as a form of manipulation (in a sense of "manipulation" stronger than in solar neutrinos, but still weaker than in the electron-gun PEGGY II).

This is not meant to be a tu quoque argument against Hacking. On the contrary, I do think that Hacking's extension of the manipulability criterion to include forms weaker than "spraying" is a perfectly consistent move. In fact, despite much emphasis on manipulation as spraying or intervening with, experimental realists are committed to a broadly construed notion of manipulation, including the entities we manipulate upon/intervene in no less than the entities we manipulate with/intervene with. And this is because manipulation/intervention is ultimately the criterion for finding out what causes what in the lab. So, in any experimental circumstance that does not allow us a "spraying"-like way of discovering what causes what (such as the one described in Section 3.2), we can still find this out by manipulating upon a target, interfering with its causal processes (e.g., by probing the quark density at different intervals with high-energy leptonic beams), and observe the experimental effects (e.g., scaling violations) that result from this intervention. Furthermore, any distinction between the active sense (manipulate with) and the passive one (manipulate upon) seems to be arbitrarily drawn in these scattering experiments, because we can interpret this same experimental scenario the other way around. Namely, it is the nucleon target, or better the inner constituents of the nucleon target that actively manipulate the electrons of the beam and make them scatter with certain scattering angles that vary depending on the dynamic properties and behavior of the nucleon's constituents.

Thus, 'seeing' quarks is not licensed by the strong notion of manipulation (spraying), but by a weaker notion of manipulation (probing) that nonetheless still satisfies the engineering criterion of experimental realism (interfering / intervening). This way of 'seeing' the nucleon's constituents presupposes a massive amount of theory. For instance, it presupposes theoretical assumptions about scattering techniques originally developed by Rutherford to probe the atomic structure. It presupposes a theory about how spectrometers work to gather and filter the scattering data from background noise and possible errors. It presupposes an interlocking chain of low level generalisations as well as recourse to fundamental laws such as momentum and energy conservation. But, it presupposes also some batches of crucial theoretical assumptions about the very same facts under investigation. Indeed, it is precisely in virtue of this last kind of theoretical 
assumptions (among others) that physicists can arguably claim to have 'seen' quarks and gluons in scattering experiments.

This remark is not meant to deny the possibility of theory-free observation in experimental physics. I have no doubt that there are fields where the notion of 'observation' gets more and more purified from any underlying theoretical assumptions, and Hacking himself has given us some interesting examples (Hacking 1983, chap. 10). Nor am I harking back to the old slogan that all observation is theory-loaded. Rather, I believe with Shapere that in certain cases what counts as 'observation' depends ultimately upon our current state of knowledge; and, most importantly for the rest of my argument, what kind of entities physicists can arguably claim to 'see' in a lab turns out to be dependent on what kind of theory about these very same entities they endorse. Both these aspects will play a major role in the discussion of the empirical equivalence between partons and quarks in what follows.

3.2. From Partons to Quarks: How to Break Down the Prima Facie Empirical Equivalence. Going then back to deep inelastic scattering experiments and to the way they opened the path to the 'observation' of the nucleon's constituents, let me introduce an important variable: the socalled scaling variable $x$

$$
x \equiv Q / 2 M v
$$

where $M$ is the proton mass, $Q^{2}$ is again the squared high-momentum transfer during the electron-proton scattering, and $\nu$ is equal to $E-E^{\prime}$ (i.e., the energy transferred in the inelastic scattering, where the initial and final energies of the scattered electrons are measured in a spectrometer). The differential cross section for inelastic electron-proton scattering to a final energy $E^{\prime}$ and scattering angle $\theta$ is

$$
\frac{d \sigma}{d E^{\prime} d \Omega}=\frac{\alpha^{2}}{4 E^{2} \sin ^{4} \frac{\theta}{2}} \frac{1}{v}\left\{F_{2}\left(x, Q^{2}\right) \cos ^{2} \frac{\theta}{2}+\frac{Q^{2}}{x M^{2}} F_{1}\left(x, Q^{2}\right) \sin ^{2} \frac{\theta}{2}\right\}
$$

where $\frac{\alpha^{2}}{4 E^{2} \sin ^{4 \theta} \frac{1}{2}}$ is the Rutherford scattering cross section, $\Omega$ is the angular distribution of scattered particles, and $F_{1}$ and $F_{2}$ are the so-called proton structure functions. They are functions of the scaling variable $x$ and $Q^{2}$, and for a fixed value of $x$-at high-momentum $Q^{2}$ - they become independent of $Q^{2}$. This crucial property of the structure functions is called Bjorken scaling or scale invariance. It means that the structure functions are invariant under a scale transformation, i.e., they remain invariant if all particle masses, energies, and momenta are multiplied by a scale factor $k$. Scaling behaviour in deep inelastic scattering was 
observed at SLAC in the late 1960s. The presence of point-like particles inside the proton was revealed precisely by the mathematical property of the functions $F$ of being independent of $Q^{2}$ at a given value of $x$. Bjorken (1969) called these particles "partons." 14

The naïve parton model assumes that there are point-like partons $i$ inside the proton carrying a charge $e_{i}$. The partons that have been struck by the incident beam are supposed to move parallel to the parent proton (i.e., they have null transverse momentum). Each parton $i$ would carry a fraction $x$ (equal to the scaling variable) of the proton's momentum $P$, and the probability that the struck parton $i$ carries a momentum fraction $x$ is given by the parton momentum distribution

$$
f_{i}(x)=\frac{d P_{i}}{d x} .
$$

The parton momentum distributions $f_{i}(x)$ are predicted to be the same at all $Q^{2}$ (i.e., they satisfy Bjorken scaling). Experimental data on the $x$-dependence of $F_{2}$ via the so-called Callan-Gross relation (Callan-Gross 1968; 1969) showed that partons were spin- $1 / 2$ particles as also quarks are (see Appendix 1).

Coming to the parton charges $e_{i}$, by combining the experimental results for the proton structure functions with those for the neutron structure functions, and those of inelastic neutrino scattering, a good agreementwithin experimental error of order $10 \%$-was found with the results for the quark charges. Let me explain this important point in more detail.

Partons lack completely the typical QCD properties of quarks, such as the ability of a struck quark to emit a gluon that in turn converts into a quark-antiquark pair belonging to the so-called sea of quark-antiquark pairs. According to QCD, the sea of quark-antiquark pairs grows logarithmically as $x \rightarrow 0$ :

$$
f_{i}(x) \underset{x \rightarrow 0}{\longrightarrow} \frac{1}{x}
$$

This means that at small $x$ values $(x \approx 0)$, the quark-antiquark sea will dominate and overshadow the so-called valence quarks (e.g., $\mathrm{u}$ and $\mathrm{d}$ quarks in the proton and neutron); vice versa, at high $x$ values $(x \approx 1)$ the valence quarks prevail over the quark-antiquark sea. This aspect plays a major role in distinguishing partons from quarks, as we will see below

14. As Bjorken pointed out in his article, the original term "parton" goes actually back to Feynman, who however published his work only three years later (Feynman 1972). Thus, the first public announcement of partons is to be found in Bjorken and Paschos (1969). 
(scaling violations). What I want to stress here is that-despite partons lacking the ability of emitting anything like gluons-still they were an empirically equivalent surrogate for quarks, and the experimental results for the parton charges $(-1 / 3$ and $2 / 3)$ turned out to be the same as those for the quark charges (see Appendix 1).

Furthermore, by using the so-called Gross-Llewellyn Smith sum rule (Gross and Llewellyn Smith 1969) on neutrino scattering, it was found that there were exactly three valence constituents inside nucleons (see Appendix 1). And once again, this was evidence for the nucleon consisting of either three quarks or three partons.

In sum, scaling behaviour tells us that there are point-like entities inside the nucleons; experimental data on the $x$-dependence of $F_{2}$ via the CallanGross relation tell us that these entities must be spin- $1 / 2$ particles; data on neutrino scattering via the Gross-Llewellyn Smith formula tell us there are exactly 3 of them in nucleons; and finally the structure function $F_{2}$ measured in combined electron and neutrino scattering tells us that these constituents have respectively charges $2 / 3$ and $-1 / 3$.

All these experimental results fit quarks and partons equally well. Yet partons and quarks are different entities endowed with different dynamic properties: partons do not radiate anything similar to gluons, and they lack completely the dynamics of colored quarks. Nonetheless, in the highmomentum region, we can freely take the results of deep inelastic scattering experiments as evidence both for partons and for quarks. Nucleons can be regarded as clusters either of partons or of quarks, and their known physical properties can be recovered in both cases. Thus, the choice as to whether partons or quarks are the basic constituents of nucleons seems to be underdetermined by experimental evidence. How did physicists then come to the conclusion that the inner constituents of nucleons were in fact quarks, and not partons?

As announced, I was playing the devil's advocate for the entity-version of empirical equivalence presented in Section 2 only to dismisss it later. The prima facie empirical equivalence between quarks and partons disappears, once we consider the measurement of the structure function $F_{2}$ in inelastic neutrino-hadron scattering in the CDHS experiment ${ }^{15}$ at CERN (de Groot et al. 1979), whose interesting results I describe below. It might seem then that experimental realists could appeal to this piece of evidence to rejoin that we do not have to fear empirical equivalence between rival entities. After all, physicists can and do discriminate between rival putative entities on a purely experimental basis. As I turn to now, however, it

15. CDHS is the acronym of a collaboration among CERN (Geneva), Dortmund, Heidelberg, and Saclay (France). 
is the emphasis on purely experimental that is highly questionable in this case.

In fact, the way the CDHS experimental result discriminated between partons and quarks was not in terms of the experimental realist's strong notion of manipulation (spraying). Given the color confinement, physicists did not know what kind of entities they were actually intervening in, while probing nucleon targets with leptonic beams. They might have been intervening in partons instead of quarks, since both entities could account for the physical properties of nucleons and experimental data about the spin and the charge of the nucleon's constituents could be equally retrieved either by assuming partons or quarks. The reasons why the CDHS experiment discriminated unequivocally between partons and quarks lay rather in the fact that while probing nucleon targets physicists were assuming batches of crucial theoretical assumptions about the nucleon's constituents. Physicists knew from QCD that quarks emit gluons, by contrast with partons, and that emitted gluons carry off some portions of the initial quark momentum. Moreover, they knew from QCD that gluons are responsible for the production of a sea of quark-antiquark pairs, and that the sea grows logarithmically when $x \rightarrow 0$ (recall eq. 4). The CDHS experimental result discriminates between partons and quarks only in the light of what QCD teaches us about quarks and their dynamic properties. In other words, in order to individuate what entity-with-causal-powers (among possibly rival and empirically equivalent ones) is at work behind the experimental results, physicists had to commit themselves to a fullblown theory (QCD) and believe in the theoretical description of the nucleon's constituents offered by this theory, rather than the one given by the rival parton model.

Notice that this does not imply that we cannot separate properties that entities have on their own (i.e., properties ascribed to the entities on purely experimental grounds) from more theoretical properties involved with an embracing theory. The story told so far clearly indicates that there were genuine entity properties: the spin- $1 / 2$ (ascribed to the nucleon's constituents by measuring the $x$-dependence of $F_{2}$ via the Callan-Gross rule), the fractional charge measured in combined electron and neutrino scattering, and the number of valence constituents measured in neutrino scattering via the Gross-Llewellyn Smith rule. These entity properties require only the aforementioned phenomenological laws of the parton model, rather than commitment to a full-blown theory such as the QCD theory of strong interaction. Yet, they are precisely the properties incriminated for being unable to distinguish partons from quarks. The additional properties we need to discriminate the nucleon's constituents are properties belonging to the broad theoretical picture of QCD (i.e., quantum-chromodynamic properties responsible for the observed scaling 
violations). So, in the end, we can and do distinguish entity properties from more theoretical ones (or better, we can distinguish an entity prototheory from the broader QCD theory). However, and this is the main point of my argument, only by endorsing the full-blown theory, can we distinguish partons from quarks and arrive at the identification of the nucleon's constituents.

In sum, we must distinguish between two different levels. At a lower/ experimental realist level (i.e., experiments plus phenomenological laws) partons and quarks are empirically equivalent. At a higher/scientific realist level (i.e., experiments plus QCD theory), partons and quarks are no longer empirically equivalent.

In fact, if we try to include quantum chromodynamic properties in the parton model, the hallmark of the model, Bjorken scaling, is violated (see Appendix 2). The violation of Bjorken scaling ${ }^{16}$ is the signal that gluons are emitted, and accordingly that colored quarks are the entities at work in deep inelastic scattering.

Scaling violations entail that the nucleon structure function $F_{2}$ does not remain invariant by changing the high-momentum transfer $Q^{2}$. Rather, the dependence of $F_{2}$ on $\log Q^{2}$ indicates that as $Q^{2}$ increases, the incident beam starts to better probe the inner structure of the nucleon. If the inner constituents were non-interacting partons, an increasing $Q^{2}$ would not resolve further structure, and Bjorken scaling would then retain its validity. But if the inner constituents are colored quarks exchanging gluons and producing an increasing sea of quark-antiquark pairs as $x$ approaches 0 , then by increasing $Q^{2}$ we should be able to probe further structure inside the nucleon. In particular, we should be able to 'see' an increasing cloud of 'soft' quarks that have lost most of their original momentum by radiating gluons. This process can be computed with the Altarelli-Parisi (1977) technique (see Appendix 2), and it involves some fundamental laws of nature: from energy conservation to momentum conservation, from the conservation of electric charge to the conservation of baryon number.

To sum up, if quarks are the entities at work in deep inelastic scattering, scaling violations must occur. What we then expect to 'observe' is that at a fixed value of the scaling variable $x$, the structure function $F_{2}$ does not remain constant by increasing $Q^{2}$. Rather, either 1 ) it decreases (at large $x$ values, i.e., $x \approx 1$ ) because the emitted gluon carries off a portion $x$ of the initial quark momentum $y$ so that the valence quark distribution shrinks towards smaller values of fractional momentum $x / y$ as $Q^{2}$ increases; or vice versa 2 ) the structure function increases (at small $x$ values, $x \approx 0$,

16. Signals of scaling violations were detected for the first time at SLAC in the mid-1970s. See Buras-Gaemers (1978). 
where the sea of quark-antiquark pairs is dominant) because the sea distribution increases by increasing $Q^{2}$. The CDHS experiment has detected precisely this type of scaling violations (see Figure 1).

At this point, an experimental realist might further retort that scaling violations are at most the experimental signal that there are additional entities inside the nucleon over and above the main constituents, be they partons or quarks. And we should content ourselves with this more modest conclusion rather than speculating on the eventual quantumchromodynamic properties of these entities. However, the question as to what these additional entities eventually would be remains unsettled. Let us suppose that experimental realists can give them also a name, possibly different from the theory-loaded "gluon." From a purely experimental point of view, we cannot even start to characterise these additional entities. Nor, needless to say, does a purely experimental situation occur in this case.

The CDHS experimental result gives us a non-equivocal characterization of these additional entities by telling us what quantum-chromo-

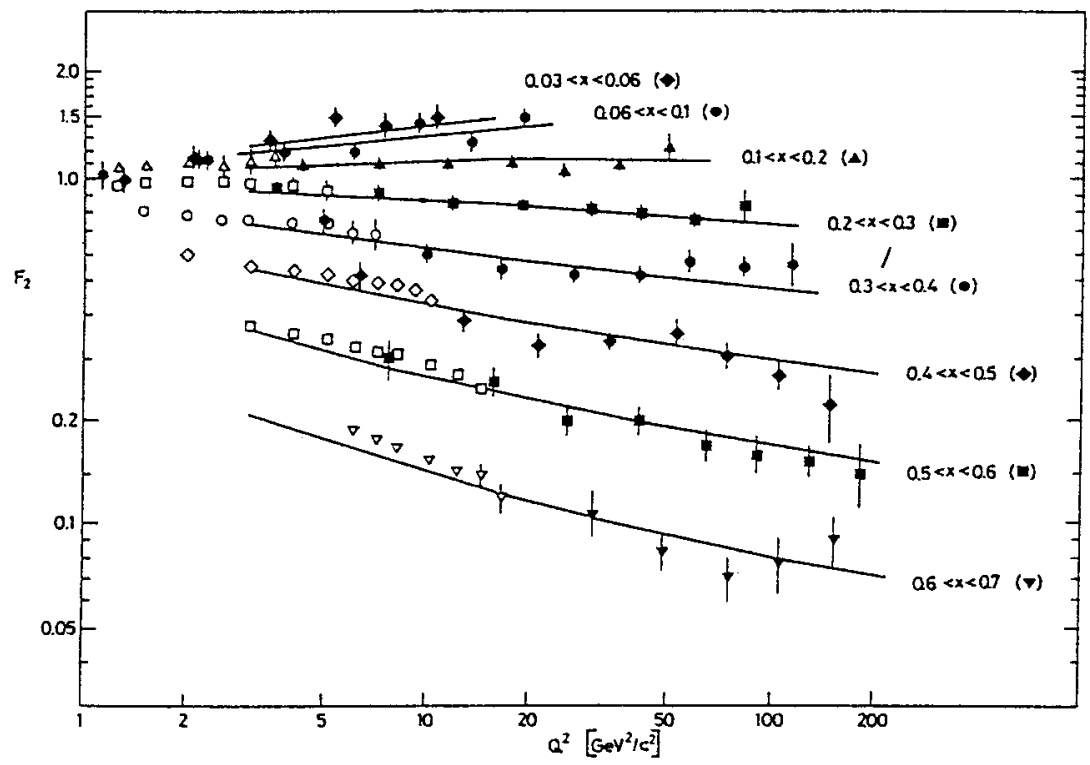

Figure 1. Scaling violations detected in the CDHS experiment. By increasing $Q^{2}$, the structure function $F^{2}\left(x, Q^{2}\right)$ either increases at small $x$ values (where the sea of quarkantiquark pairs is dominant), or it decreases at large $x$ (where the valence quarks are dominant, and emit gluons carrying off portions of the parent quark momentum) (de Groot et al. 1979). 
dynamic properties (causal powers/capacities/dispositions) they must have for scaling violations of the observed type in Figure 1 to occur. Namely, these additional entities must be radiated by the nucleon constituents, they must carry off a fraction of the nucleon constituent's momentum $y$ as computed by the Altarelli-Parisi equation, for $F^{2}$ to be detected to decrease at large $x$ values, and to increase at small $x$ values. Scaling violations are the experimental signal of the existence of additional particles inside nucleons only insofar as they are-in the first place - the signal that the QCD picture of nucleons is correct. Echoing Resnik, experimental realists can only have knowledge about theoretical entities if they assume that the theory describing those entities is at least approximately true.

Experimenters ended up believing in quarks because they believed the dynamic picture offered by QCD. Experimental evidence can give the verdict to one kind of entity rather than to another only because it has already given the verdict to one scientific model rather than another: the entity-version of empirical equivalence can be solved only by solving the theory-version.

4. Conclusion. Scaling violations are a decisive testing ground to resolve the prima facie empirical equivalence between partons and quarks as rival potential constituents of nucleons. If we take partons and quarks simply as putative entities composing nucleons regardless of any theoretical commitment about what partons and quarks are respectively up to (as experimental realism claims), it seems that we have no more reasons for believing in quarks than for believing in partons. But this is not the case. We do have more reasons for believing in quarks once we start looking at the dynamic properties of partons and quarks as they are given by the parton model and QCD, respectively. Hardly ever can we take an entity apart from its physical properties: these are encoded by the relevant theory, and we cannot believe in the existence of an entity endowed with these properties without also believing as correct the theory that characterises the entity as such.

Nor is this theoretical commitment confined to low-level phenomenological laws. Once we accept the QCD picture, we accept its entire theoretical arsenal including fundamental laws as well as phenomenological ones.

The middle ground of experimental realism turns out to be a nondefensible option when it comes to justifying our belief in quarks. The experimental evidence for them is highly theoretical, and if experiments license realism about quarks at all, they license realism also about the scientific theory featuring them. 


\section{Appendix}

Appendix 1. Partons' Spin, Charges, and Number Inside Nucleons

The structure function $F_{2}$ is the same both in the case that partons were spin-0 or spin- $1 / 2$ particles, namely

$$
F_{2}\left(x, Q^{2}\right)=\sum_{i} e_{1}^{2} x f_{i}(x)
$$

where $e_{i}$ are the parton charges and $f_{i}$ the parton momentum distribution.

By contrast, the structure function $F_{1}$ depends on the spin of the partons: if partons were spin-0 particles it would be

$$
F_{1}\left(x, Q^{2}\right)=0
$$

whereas if partons were spin- $1 / 2$ particles it would be

$$
F_{1}\left(x, Q^{2}\right)=\frac{1}{2 x} F_{2}\left(x, Q^{2}\right)
$$

where (7) is the Callan-Gross relation mentioned in Section 3.2. By measuring the $x$-dependence of $F_{2}$ and by using this phenomenological law, it was found that partons could not be spin-0 particles. They were spin-1/2 particles like quarks.

Coming to the electric charges, the empirical equivalence between partons and quarks emerged from combined neutrino and electron scattering experiments. Let us for simplicity consider only scattering experiments in the region of $x \geq 0.3$ where, as mentioned in Section 3.2, the QCD sea of quark-antiquark pairs can be neglected to a good approximation, while valence quark distributions prevail. In this region, the only non-vanishing quark distributions for the proton are $u^{\mathrm{p}}(x)$ and $d^{p}(x)$. Instead of speaking of $\mathrm{u}$ and d quark, in order to stick to parton language, let us rather speak in terms of partons $i_{1}$ and $i_{2}$, and parton distributions $f_{i_{1}}(x)$ and $f_{i_{2}}(x)$. The proton structure function measured in inelastic electron scattering in the region of $x \geq 0.3$ is

$$
F_{2}^{e p}(x)=e_{i_{1}}{ }^{2} x f_{i_{1}}(x)+e_{i_{2}}{ }^{2} x f_{i_{2}}(x) .
$$

The corresponding result for inelastic neutrino-proton scattering is

$$
F_{2}^{v p}(x)=x f_{i_{2}}(x)
$$


Since proton and neutron belong to the same isospin doublet (in QCD language, $\mathrm{p}=$ uud and $\mathrm{n}=\mathrm{udd}$ ) their parton distributions are related as follows

$$
\begin{aligned}
& i_{1}^{p}(x)=i_{2}^{n}(x) \equiv i_{1}(x) \\
& i_{2}^{p}(x)=i_{1}^{n}(x) \equiv i_{2}(x)
\end{aligned}
$$

Accordingly, the neutron structure function measured in inelastic electron scattering in the region of $x \geq 0.3$ reduces to

$$
F_{2}{ }^{e n}(x)=e_{i_{1}}{ }^{2} x f_{i_{2}(x)}+e_{i_{2}}{ }^{2} x f_{i_{1}}(x)
$$

while the corresponding structure function for neutrino scattering is

$$
F_{2}{ }^{v n}(x)=x f_{i_{1}}(x)
$$

Thus, by combining the results of electron scattering and neutrino scattering for protons and neutrons we obtain (see Martin and Shaw 1999, § 7.5.1)

$$
\frac{F_{2}^{e p}(x)+F_{2}^{e n}(x)}{F_{2}^{v p}(x)+F_{2}^{v n}(x)}=\frac{e_{i_{1}}{ }^{2}+e_{i_{2}}{ }^{2}}{2}=\frac{5}{18} .
$$

In other words, the nucleon $N$ structure function $F_{2}{ }^{e N}$ measured in electron scattering is equal to $5 / 18$ the corresponding structure function measured in neutrino scattering, i.e., $0.27 F_{2}{ }^{v N}$ and, indeed the experimental result for this ratio turned out to be $0.29 \pm 0.02$.

The factor $5 / 18$ comes from mean squared charges $\frac{1}{2}\left[\left(-\frac{1}{3}\right)^{2}+\left(\frac{2}{3}\right)^{2}\right]$ of the nucleon constituents. And this experimental result can be interpreted as evidence both for charged partons as well as for charged quarks.

Moreover, if we neglect once again the sea components and integrate over the valence constituents with normalisation

$$
\int_{0}^{1} f_{i_{1}}(x) d x=2 \quad \int_{0}^{1} f_{i_{2}}(x) d x=1
$$

by using the Gross-Llewellyn Smith sum rule on neutrino scattering

$$
\int_{0}^{1} F_{3}{ }^{v N}(x) d x=\int_{0}^{1}\left[f_{i_{1}}(x)+f_{i_{2}}(x)\right] d x=3
$$

we can also count the number of valence constituents inside the nucleon: there are exactly three of them, as in the case of quarks. 


\section{Appendix 2. Bjorken Scaling Violations}

If we include gluon effects, the nucleon structure function $F_{2}$ of the parton model must be rewritten as

$$
\frac{F_{2}\left(x, Q^{2}\right)}{x}=\sum_{q} e_{q}^{2}\left(q(x)+\Delta q\left(x, Q^{2}\right)\right)
$$

where the parton distribution function $f_{i}(x)$ has been replaced by the quark density $q(x)$, and $\Delta q$ is equal to

$$
\Delta q\left(x, Q^{2}\right) \equiv \frac{\alpha_{s}}{2 \pi} \log \left(\frac{Q^{2}}{\mu^{2}}\right) \int_{x}^{1} \frac{d y}{y} q(y) P_{q q}\left(\frac{x}{y}\right)
$$

where $P_{q q}\left(\frac{x}{y}\right)$ is the probability of a quark with momentum fraction $y$ emitting a gluon, and hence becoming a quark with momentum fraction $x / y$. The new structure function (16) is dependent both on $x$ and on $Q^{2}$, which varies logarithmically: thus, it violates Bjorken scaling.

By integrating over all momentum fractions $y$ (where $y>x$ ) of the parent nucleon, there is a probability proportional to $\alpha_{s} P_{q q}(x / y)$ that a quark with momentum fraction $x$ has originated from a previous quark with momentum fraction $y$ of the parent nucleon that has lost part of its momentum by radiating gluons (see Halzen and Martin 1984, $\S 10.5$ ). The Altarelli-Parisi evolution equation controls this process by probing different intervals of the quark density:

$$
\frac{d}{d \log Q^{2}} q\left(x, Q^{2}\right)=\frac{\alpha_{s}}{2 \pi} \int_{x}^{1} \frac{d y}{y} q\left(y, Q^{2}\right) P_{q q}\left(\frac{x}{y}\right) .
$$

This technique presupposes that the momentum, the mass, the baryon number and the charge of the parent nucleon are conserved in the aforementioned QCD interactions among the constituent quarks.

\section{REFERENCES}

Altarelli, G. and G. Parisi (1977), "Asymptotic Freedom in Parton Language”, Nuclear Physics B 126: 298-318.

Bjorken, J. D., and E. A. Paschos (1969), "Inelastic Electron-Proton and $\gamma$-Proton Scattering and the Structure of Nucleon", Physical Review 185: 1975-1982.

Buras, A. J., and K. J. F. Gaemers (1978), "Simple Parametrization of Parton Distributions with $Q^{2}$ Dependence Given by Asymptotic Freedom", Nuclear Physics B 132: 249-267.

Callan, C. G., and D. J. Gross (1968), "Crucial Test of a Theory of Currents", Physical Review Letters 21: 311-313. (1969), "High-energy Electroproduction and the Constitution of Electric Current", Physical Review Letters 22: 156-159.

Cartwright, Nancy (1983), How the Laws of Physics Lie. Oxford: Oxford University Press. 
Clarke, Steve (2001), "Defensible Territory for Entity Realism", British Journal for the Philosophy of Science 52: 701-722.

Close, F. E. (1997), "Glueballs and Hybrids: New States of Matter", Contemporary Physics 38: $1-12$.

de Groot, J. G. H., et al. (1979), "Inclusive Interactions of High-Energy Neutrinos and Antineutrinos in Iron", Zeitschrift für Physik C, Particles and Fields 1: 143-162.

Feynman, Richard P. (1972), Photon-hadron Interactions. New York: Benjamin.

Franklin, Alan (1996), "There Are No Antirealists in the Laboratory", in R. S. Cohen, R. Hilpinen, and Q. Renzong (eds.), Realism and Anti-Realism in the Philosophy of Science. Dordrecht: Kluwer, 131-148.

Gell-Mann, Murray (1961), "The Eightfold Way: a Theory of Strong Interaction Symmetry", California Institute of Technology Synchrotron Laboratory Report CTSL-20. Reprinted in Murray Gell-Mann and Yuval Ne'eman (eds.), The Eightfold Way. New York: W. A. Benjamin, 1964, 11-57.

-(1964), “A Schematic Model of Baryons and Mesons", Physics Letters 8: 214-15. Reprinted in Murray Gell-Mann and Yuval Ne'eman (eds.), The Eightfold Way. New York: W. A. Benjamin, 1964, 168-169.

Gross, D. J., and C. H. Llewellyn Smith (1969), "High-energy Neutrino-nucleon Scattering, Current Algebra and Partons", Nuclear Physics B 14: 337-347.

Hacking, Ian (1982), "Experimentation and Scientific Realism", Philosophical Topics 13: $71-87$.

(1983), Representing and Intervening. Cambridge: Cambridge University Press.

Halzen, Francis, and Alan D. Martin (1984), Quarks and Leptons: An Introductory Course in Modern Particle Physics. New York: John Wiley \& Sons.

Han, M. Y., and Y. Nambu (1965), "Three-Triplet Model with Double SU(3) Symmetry", Physical Review B 139: 1006-1010.

Kukla, André (1996), “Does Every Theory Have Empirically Equivalent Rivals?”, Erkenntnis 44: $137-166$.

Laudan, Larry (1990), "Demystifying Underdetermination", in C. Wade Savage (ed.), Scientific Theories. Minnesota Studies in the Philosophy of Science, vol. 14. Minneapolis: University of Minnesota Press, 267-297.

Laudan, Larry, and Jarrett Leplin (1991), "Empirical Equivalence and Underdetermination”, Journal of Philosophy 88 (9): 449-472.

Massimi, Michela (forthcoming), "What Demonstrative Induction Can Do against the Threat of Underdetermination: Bohr, Heisenberg, and Pauli on Spectroscopic Anomalies (1921-24)", Synthese.

Martin, B. R., and G. Shaw (1999) Particle Physics, 2d ed. Chichester: John Wiley \& Sons.

Musgrave, Alan (1996), "Realism, Truth, and Objectivity", in R. S. Cohen, R. Hilpinen and Q. Renzong (eds.), Realism and Anti-realism in the Philosophy of Science. Dordrecht: Kluwer, 19-44.

Ne'eman, Yuval (1961), "Derivation of Strong Interactions from a Gauge Invariance", Nuclear Physics 26: 222-229. Reprinted in Murray Gell-Mann and Yuval Ne'eman (eds.), The Eightfold Way. New York: W. A. Benjamin, 1964, 58-65.

Putnam, Hilary (1975), "The Meaning of 'Meaning",, in K. Gunderson (ed.), Language, Mind and Knowledge. Minnesota Studies in the Philosophy of Science, vol. 7. Minneapolis: University of Minnesota Press. Reprinted in H. Putnam, Mind, Language and Reality. Philosophical Papers, vol. 2. Cambridge: Cambridge University Press, $1975,215-271$.

Resnik, David B. (1994), “Hacking's Experimental Realism”, Canadian Journal of Philosophy 24: 395-412.

Sellars, Wilfred (1962), Science, Perception, and Reality. New York: Humanities Press.

Shapere, Dudley (1982), "The Concept of Observation in Science and Philosophy", Philosophy of Science 49: 485-525.

(1993), "Astronomy and Anti-Realism", Philosophy of Science 60: 134-50.

van Fraassen, Bas (1980), The Scientific Image. Oxford: Oxford University Press. 